\title{
旱区城市扩展过程区位因素研究 一以中国呼包鄂榆城市群为例
}

\author{
宋世雄 ${ }^{1,2}$, 张金茜 ${ }^{1,2}$, 刘志锋 ${ }^{1,2}$, 何春阳 ${ }^{1,2}$ \\ (1. 北京师范大学地表过程与资源生态国家重点实验室, 人与环境系统可持续研究中心, 北京 100875; \\ 2. 北京师范大学地理科学学部自然资源学院, 土地资源与区域发展研究中心, 北京 100875)
}

\begin{abstract}
摘要:认识城市扩展过程的区位因素特征对旱区城市可持续发展具有重要意义。为此, 以中国 呼包鄂榆城市群为例, 利用随机森林方法量化区位因素对城市扩展过程的影响。研究发现: 随 机森林方法能够有效地量化旱区城市扩展过程区位因素的基本特征, 模型的 AUC ( area under curve) 值达到 0.97。同时, 到城市中心距离对区域 1980-2017年城市扩展过程影响最大, 重要 性达到 $42.62 \%$ 。国道、高速公路和铁路等交通因素也有重要的影响, 重要性均大于 $10 \%$ 。此 外,所有区位因素对区域城市扩展过程的影响均存在尺度效应,其中地形、气候和河流对城市 扩展过程影响的尺度效应相对比较明显。地形、气候和河流对大城市影响的重要性分别为 $27.17 \% 、 20.23 \%$ 和 $8.12 \%$, 分别是其对小城市影响的 4.02 倍、3.91倍和 2.36 倍。因此, 建议在旱 区城市建设中, 应该高度重视地形、气候和河流等自然要素的约束作用,因地制宜地进行城市 规划和建设。
\end{abstract}

关键词: 随机森林; 区位因素;城市扩展过程;旱区;呼包鄂榆城市群

旱区是指多年平均降水量与多年平均潜在蒸散量之比小于 0.65 的地区 ${ }^{[1]}$ 。全球有 $38 \%$ 的人口生活在旱区，该地区具有生态环境脆弱、城市化迅速以及对气候变化敏感等特 点, 是全球可持续发展的关键区域之一“]。城市扩展过程是指城市化进程中城市土地的增 长过程 ${ }^{[2]}$, 它在提升人类福祉的同时，也为区域环境带来严重的影响 ${ }^{[3-5]}$ 。交通、地形和 位置等区位因素能够通过影响城市扩展的空间格局，进而改变城市扩展对区域环境的影 响 ${ }^{[6-8]}$ 。近年来，伴随着快速的社会经济发展，旱区经历了快速的城市扩展过程 ${ }^{[9,10]}$, 这一 过程对区域的自然生境 ${ }^{[1]]}$ 、生物多样性 ${ }^{[12]}$ 以及环境可持续性 ${ }^{[3]}$ 等产生了严重影响。因此, 充分认识城市扩展过程的区位因素特征，对于缓解旱区的环境压力以及实现区域城市的 可持续发展具有重要意义。

目前, 已有研究通过定性或定量方法来揭示旱区城市扩展过程的区位因素特征。定 性分析方面，潮洛濛等 ${ }^{[13]}$ 分析了区位因素对呼和浩特市 1987-2006年城市扩展过程的影 响。李寒国等 ${ }^{[14]}$ 分析了影响张掖市 1977-2010年城市扩展过程的区位因素。定量分析方 面，黄庆旭等 ${ }^{[15]}$ 利用 Logistic 回归方法分析了坡度、高程和交通等区位因素对北京市 1991-2004 年城市扩展的影响。Shafizadeh-Moghadam 等 ${ }^{[16}$ 使用地理加权 Logistic 回归方

收稿日期：2019-11-08; 修订日期：2020-05-27

基金项目：国家自然科学基金项目（41971270，41971271)

作者简介：宋世雄（1990- ), 男，陕西乾县人，博士研究生，主要从事城市扩展过程模拟及其影响研究。 E-mail: ssx1990@126.com

通讯作者: 刘志锋 (1986-), 男, 江西景德镇人, 博士, 副教授, 主要从事景观地理与景观可持续科学研究。 E-mail: Zhifeng.Liu@bnu.edu.cn 
法分析了印度孟买 1973-2010年城市扩展的区位因素。Wu 等 ${ }^{[17]}$ 通过Logistic 回归方法探 索区位因素对京津冀城市群 1980-2010年城市扩展的影响。可以看出，现有研究主要通 过Logistic 回归方法定量分析旱区城市扩展过程的区位因素特征。然而由于Logistic 回归 方法不能很好拟合非线性问题并且结果会受到多元共线性的影响，从而导致对旱区城市 扩展过程区位因素特征的认识不够准确。

随机森林是一种机器学习算法，它能够很好地拟合非线性关系，而且拟合结果不会 受到多元共线性的影响，同时可以评估特征因素的重要性 ${ }^{[18]}$ 。当前，随机森林已被应用 于城市土地利用变化区位因素研究。如Kamusoko 等 ${ }^{\left[{ }^{[1]}\right.}$ 利用随机森林分析了影响津巴布韦 Harare 地区城市土地扩展的主要区位因素。Zhang 等 ${ }^{[20]}$ 利用随机森林分析了中国惠州城市 内部土地利用变化区位因素的特征。但利用随机森林方法在旱区开展的相关研究仍比 较少。

鉴于此，本文的目的是利用随机森林方法分析早区城市扩展过程的区位因素特征。 首先，以中国呼包鄂榆城市群为例，量化区域 1980-2017年城市扩展过程的时空格局。 然后，利用随机森林方法分析影响区域城市扩展过程的区位因素。最后，根据区位因素 的特征，为区域未来城市扩展提出相应的政策建议，以期为区域可持续发展提供帮助。

\section{1 研究方法与数据来源}

\section{1 研究区概况}

呼包鄂榆（Hohhot-Baotou-OrdosYulin, HBOY）城市群位于中国北方 早区中部，在 $36^{\circ} 48^{\prime} 50^{\prime \prime} \sim 42^{\circ} 44^{\prime} 5^{\prime \prime} \mathrm{N}$ 、 $106^{\circ} 28^{\prime} 16^{\prime \prime} \sim 122^{\circ} 18^{\prime} 7^{\prime \prime} \mathrm{E}$ 之间，总面积 为 17.46 万 $\mathrm{km}^{2}$ (图 1)。该地区平均 海拔约 $1300 \mathrm{~m}$ 。气候类型为温带大 陆性季风气候，多年平均气温约为 $8{ }^{\circ} \mathrm{C}$, 多年平均降水量约为 $320 \mathrm{~mm}^{[2]}$ 。 根据 2017 年的人口数据, 参考 Huang 等 ${ }^{[22]}$ 的方法, 可将区域内 30 个城市划 分为 2 个大城市 (总人口在 100 万 500 万之间）、2个中等城市（总人口 在 50 万 100 万之间）和 26 个小城市 (总人口小于 50 万)。

20 世纪 80 年代以来, 呼包鄂榆 城市群经历了快速的城市扩展过程。 区域生产总值从 1980 年的 25.32 亿元 增长到 2017 年的 13722.25 亿元，年 均增长 370.19 亿元, 年均增长率为 $14.62 \%$ 。其中，第二产业和第三产业 分别由 12.62 亿元和 6.07 亿元增长到

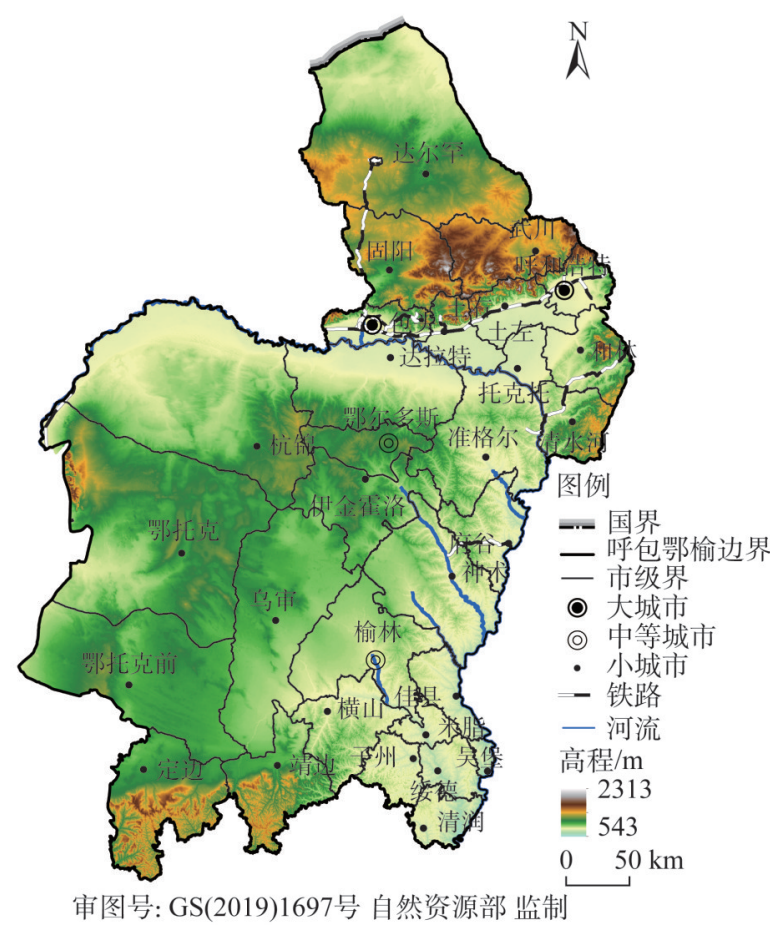

注: 本图基于自然资源部标准地图服务系统下载的标准地图 制作，底图无修改，下同。

\section{图 1 研究区概况}

Fig. 1 The study area (Huhhot-Baotou-Ordos-Yulin, HBOY for short) 
6635.86 亿元和 6600.76 亿元, 年均增长率分别为 $14.18 \%$ 和 $29.36 \%^{[23-25]}$ 。该地区非农人口 从 1990 年的 239.87 万人增加到 2010 年的 462.13 万人, 增长了 $92.66 \%^{[26]}$ 。城市土地面积从 1990 年的 $151.29 \mathrm{~km}^{2}$ 增加到了 2017 年的 $1230.86 \mathrm{~km}^{2}$, 增长了 8.14 倍 ${ }^{[27]}$ 。途经研究区的高 速公路主要有北京一拉萨高速公路、荣成一乌海高速公路和包头一茂名高速公路, 国道 主要包括 109 和 110 国道, 铁路主要有包头一兰州、包头一西安和北京一包头等铁路线, 还包括呼和浩特一包头高铁和呼和浩特一鄂尔多斯动车等。未来, 区域将建设呼和浩特 一准格尔一鄂尔多斯、准格尔一朔州、蒙西一华中和神木一瓦塘等铁路，建设保德—榆 林、呼和浩特一朔州、赛罕塔拉一二连浩特等高速公路 ${ }^{[23]}$ 。

\section{2 数据来源}

本文使用的数据主要包括 Landsat遥感影像、数字高程模型（Digital Elevation Mod$\mathrm{el}, \mathrm{DEM}) 、 气$ 象数据和地理信息辅助数据。Landsat 遥感影像来源于美国地质调查局 (https://earthexplorer.usgs.gov/)。其中，1980年的影像数据来源于 Landsat MSS 传感器, 空间分辨率为 $60 \mathrm{~m} ; 1990$ 年、2000年、2010年和 2017 年的影像数据来源于 Landsat TM/ OLI 传感器, 空间分辨率为 $30 \mathrm{~m}$ 。DEM数据来源于美国太空总署（NASA）和国防部国 家测绘局（NIMA）发布的SRTM（Shuttle Radar Topography Mission）DEM数据（http:// srtm.csi.cgiar.org/SELECTION/inputCoord.asp), 空间分辨率为 $90 \mathrm{~m}$ 。研究区周边 29 个气 象观测站数据来自于中国气象数据网（http://data.cma.cn/site/index.html）, 主要包括气温 和相对湿度数据。降雨数量来自中国科学院资源环境数据云平台（http://www.resdc.cn/data.aspx?DATAID=229)。基础地理信息数据包括研究区的行政边界、县级驻地和河流等, 数据来源于国家基础地理信息中心（http://ngcc.sbsm.gov.cn/）。2000年、2009年和2016年 的高速公路、铁路、国道等交通数据来源于北京大学城市与环境学院地理数据平台 (http://geodata.pku.edu.cn)。区域 1990 年交通数 据是基于已有交通数据结合谷歌地球高分影像 目视解译获取。为了保证数据一致性, 所有数 据采用统一的Albers 投影, 并重采样为 $30 \mathrm{~m}$ 。

\section{3 研究方法}

\subsection{1 提取区域 1980-2017年城市土地信息}

基于 Google Earth Engine 平台在线获取 Landsat MSS/TM/OLI 影像, 并通过目视解译 提取呼包鄂榆城市群 1980-2017年城市土地信 息（图2）。主要包括以下三个步骤：首先，提 取潜在的城市土地信息。参考 Liu 等 ${ }^{[28]}$ 的研究, 基于预处理后的 Landsat 影像分别计算归一化植 被指数 (Normalized Difference Vegetation Index, NDVI）、归一化建筑指数（Normalized Difference Built-up Index, NDBI) 和归一化水 体指数 (Normalized Difference Water Index, NDWI）指数, 然后采用阈值法提取该区域潜 在的城市土地信息。具体公式如下:

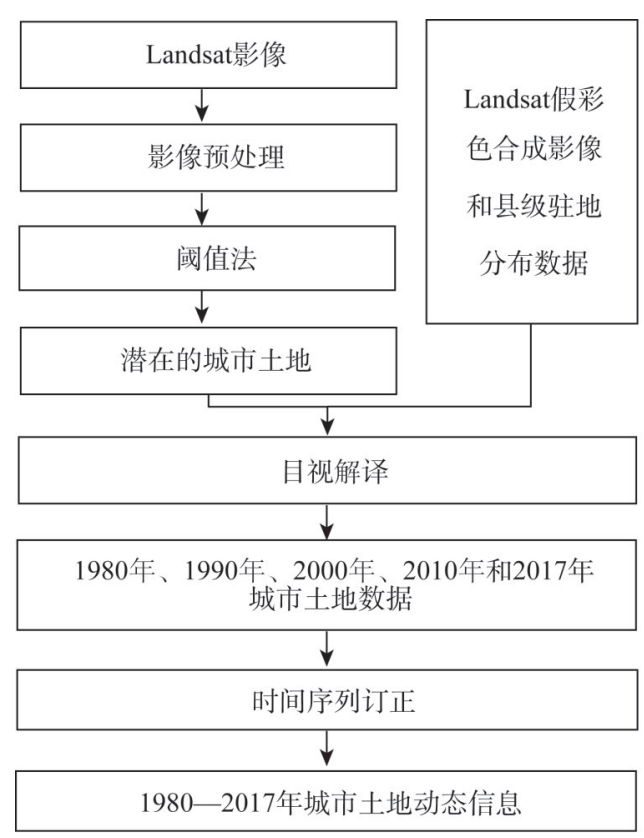

图 2 区域近四十年城市土地提取流程

Fig. 2 The flow chart for extracting urban land of the HBOY from 1980 to 2017 


$$
U L_{(n, i)}=\left\{\begin{array}{l}
1,\left(N D V I_{(n, i)} \leqslant 0.15\right) \&\left(N D B I_{(n, i)} \geqslant-0.1\right) \&\left(N D W I_{(n, i)} \leqslant 0.05\right) \\
0, \text { else }
\end{array}\right.
$$

式中: $U L_{(n, i)}$ 表示第 $i$ 个像元第 $n$ 年是否为潜在城市, 1 表示潜在城市, 0 表示非潜在城 市; $N D V I_{(n, i)}$ 表示第 $i$ 个像元第 $n$ 年的 $N D V I$ 值; $N D W I_{(n, i)}$ 表示第 $i$ 个像元第 $n$ 年的 $N D W I$ 值; $N D B I_{(n, i)}$ 表示第 $i$ 个像元第 $n$ 年的 $N D B I$ 值。其次，获取 1980 年、1990年、2000年、 2010 年和 2017 年的城市土地数据。在潜在城市土地信息基础上, 结合 Landsat 假彩色影 像和县级驻地分布数据, 进行目视解译获取城市土地数据。最后, 获取 1980-2017年城 市土地动态信息。参考 $\mathrm{He}$ 等 ${ }^{[1]}$ 的研究，对城市土地数据进行时间序列订正。时间序列订 正的基本假设是城市土地为持续增长的，通过订正可得到区域 1980-2017年的城市土地 动态信息。具体公式如下:

$$
U L_{(n, i)}=\left\{\begin{array}{lc}
0 & U L_{(n+1, i)}=0 \\
1 & U L_{(n+1, i)}=1 \& U L_{(n-1, i)}=1 \\
U L_{(n, i)} & \text { o th erwise }
\end{array}\right.
$$

式中: $U L_{(n, i)}$ 、 $U L_{(n+1, i)}$ 和 $U L_{(n-1, i)}$ 分别表示第 $i$ 个像元第 $n$ 年、第 $n+1$ 年和第 $n-1$ 年是否 为城市, 1 表示城市, 0 表示非城市。

\subsection{2 分析区域 1980 - 2017 年城市扩展过程}

参照董宁等 ${ }^{\left[{ }^{[2]}\right.}$ 的研究, 选择斑块密度（Patch density, PD）、景观形状指数（Landscape shape index, LSI) 和景观扩展指数（Landscape expansion index, LEI) 等三个指 标来表征城市景观的破碎度和形状以及城市扩展模式。其中 $P D$ 的计算公式如下:

$$
P D=N / A
$$

式中: $N$ 表示景观中斑块的总数 (个); $A$ 为景观总面积 $\left(\mathrm{hm}^{2}\right) 。 P D$ 值越大, 表示斑块的 破碎化程度越高。

$L S I$ 的计算公式如下:

$$
L S I=0.25 E / \sqrt{A}
$$

式中: $E$ 表示景观中斑块边界的总长度 $(\mathrm{m}) ; A$ 为景观总面积 $\left(\mathrm{hm}^{2}\right)$ 。 $L S I$ 值越大, 表示 斑块的形状越不规则。

$L E I$ 的计算公式如下:

$$
L E I=\frac{A_{o}}{A_{o}+A_{v}} \times 100 \%
$$

式中： $A_{o}$ 表示新增城市用地的缓冲区与已有城市用地相交的面积 $\left(\mathrm{m}^{2}\right) ; A_{v}$ 表示该缓冲 区与非城市用地相交的面积 $\left(\mathrm{m}^{2}\right)$ 。参考 $\mathrm{Liu}$ 等 ${ }^{[30]}$ 的研究, 新增城市用地缓冲区设置为 $1 \mathrm{~m}$ 。 根据 $L E I$ 值的不同, 可将城市扩展模式分成蛙跃型、边缘型和内填型三种类型 ${ }^{[30]}$ 。蛙跃型 是指新增的城市土地与已有城市土地之间没有直接接触的扩展模式, 即 $L E I=0$ 。边缘型 是指在已有城市土地的边缘继续扩展的模式, 即 $L E I$ 值在 $0 \sim 50$ 之间。内填型是指新增城 市土地被已有城市土地包围的区域的扩展模式，即 $L E I$ 在 $50 \sim 100$ 之间 ${ }^{[30]}$ 。

\subsection{3 分析区域 1980-2017年城市扩展过程的区位因素}

随机森林模型可以通过计算袋外数据错误率来得到因素的重要性评分 ${ }^{[18,20]}$ 。首先，随 机森林模型通过有放回的随机抽样法在原始数据中随机选择部分数据作为样本数据集, 
未被选择的数据成为袋外数据。然后, 针对样本数据进行多个决策树建模, 并通过众数 投票的方式得到样本数据的训练结果。最后, 通过与袋外数据进行比较, 计算模型的预 测错误率来得到影响因素的重要性评分。

参考相关研究 ${ }^{[13,15,3,1,32]}$ 以及数据的 可获取性，首先从自然和人文两个方 面选择了 9 个影响区域城市扩展过程 的区位因素，分别是高程、坡度、坡 向、到河流（主要河流）距离、干燥 度、到高速公路距离、到铁路距离、 到国道距离和到城市中心距离, 并使 用ArcGIS 10.6 进行空间化（表 1)。 然后，通过四个步骤构建随机森林模 型以分析区位因素对城市扩展过程的 影响（图 3)。第一，随机选择 $66 \%$ 的 区位因素数据为样本数据集, $34 \%$ 为 袋外数据。第二，构建 100 个决策树

\section{表 1 区位因素选取及其空间化方法}

Table 1 The location factors and spatialization methods

\begin{tabular}{cll}
\hline 类别 & \multicolumn{1}{c}{ 指标 } & \multicolumn{1}{c}{ 空间化方法 } \\
\hline 自然因素 & 高程 & 基于 DEM数据, 利用Arc- \\
& 坡度 & GIS 的地表分析模型进行 \\
& 坡向 & 计算坡度和坡向 ; 利用 \\
& 到河流距离 & ArcGIS 的距离分析模型计 \\
& 干燥度 & 参到河流距离 \\
& & 平均关研究 ${ }^{[1]}$, 利用多年 \\
& & 在蒸散量之比计算 \\
& & 利用ArcGIS 的距离分析模 \\
人文因素 & 到城市中心距离 \\
& 到高速公路距离 & 型计算到城市中心、高速 \\
& 到铁路距离 & 公路、铁路和国道距离 \\
& 到国道距离 & \\
\hline
\end{tabular}
对样本数据集进行训练, 并与袋外数据进行对比计算袋外得分, 以此评估模型的精度。 一般得分越高代表模型精度越好, 在本文中, 袋外得分大于 0.95 时认为模型精度符合要 求。第三, 利用袋外数据与每个决策树计算误差 $\left(\mathrm{e}_{1}\right)$, 然后随机调换袋外数据中的某一 个因素 $j$ 的顺序得到新的袋外数据, 并再次计算误差 $\left(\mathrm{e}_{2}\right)$ 。第四, 将每个决策树 $\mathrm{e}_{1}$ 和 $\mathrm{e}_{2}$ 的 差值标准化后求均值即可得到因素 $j$ 的重要性。本文将因素的重要性得分作为判断某一因 素对城市扩展过程影响程度的指标，得分越高说明该因素对城市扩展过程的影响越大。

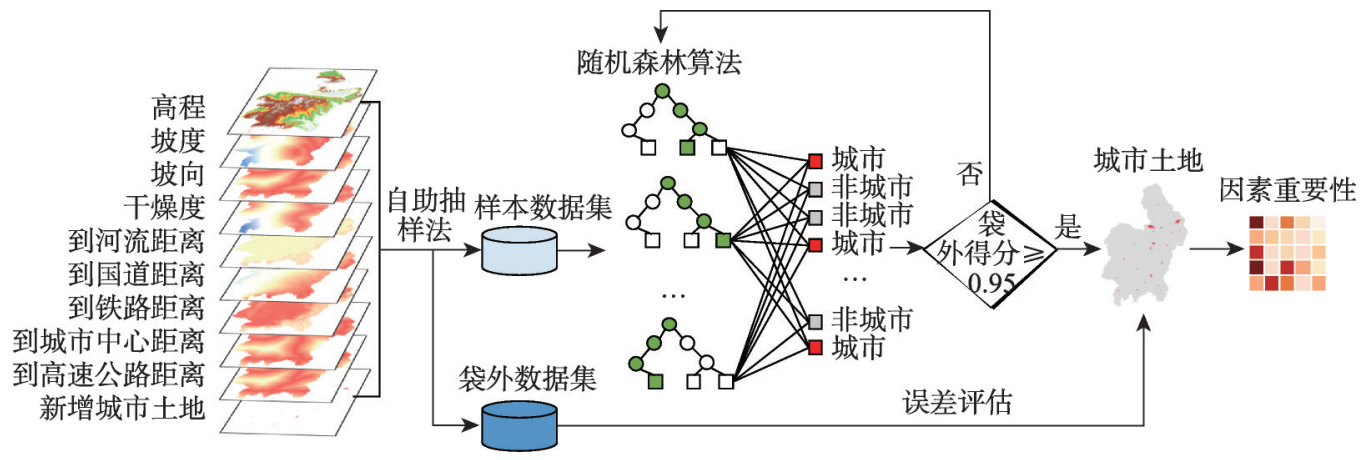

图 3 随机森林结构

Fig. 3 The structure of random forest

\section{2 结果分析}

\section{1 区域城市扩展过程}

区域1980-2017年经历了快速的城市扩展过程（图4）。全区城市土地面积由 $81.57 \mathrm{~km}^{2}$ 增加到 $1231.33 \mathrm{~km}^{2}$, 年均增长率为 7.61\%（图 5a、表 2)。区域 1980-2017年新增城市土 地主要分布在大城市, 大城市新增城市土地占全区扩展总面积的 $49.10 \%$ 。在大城市中, 


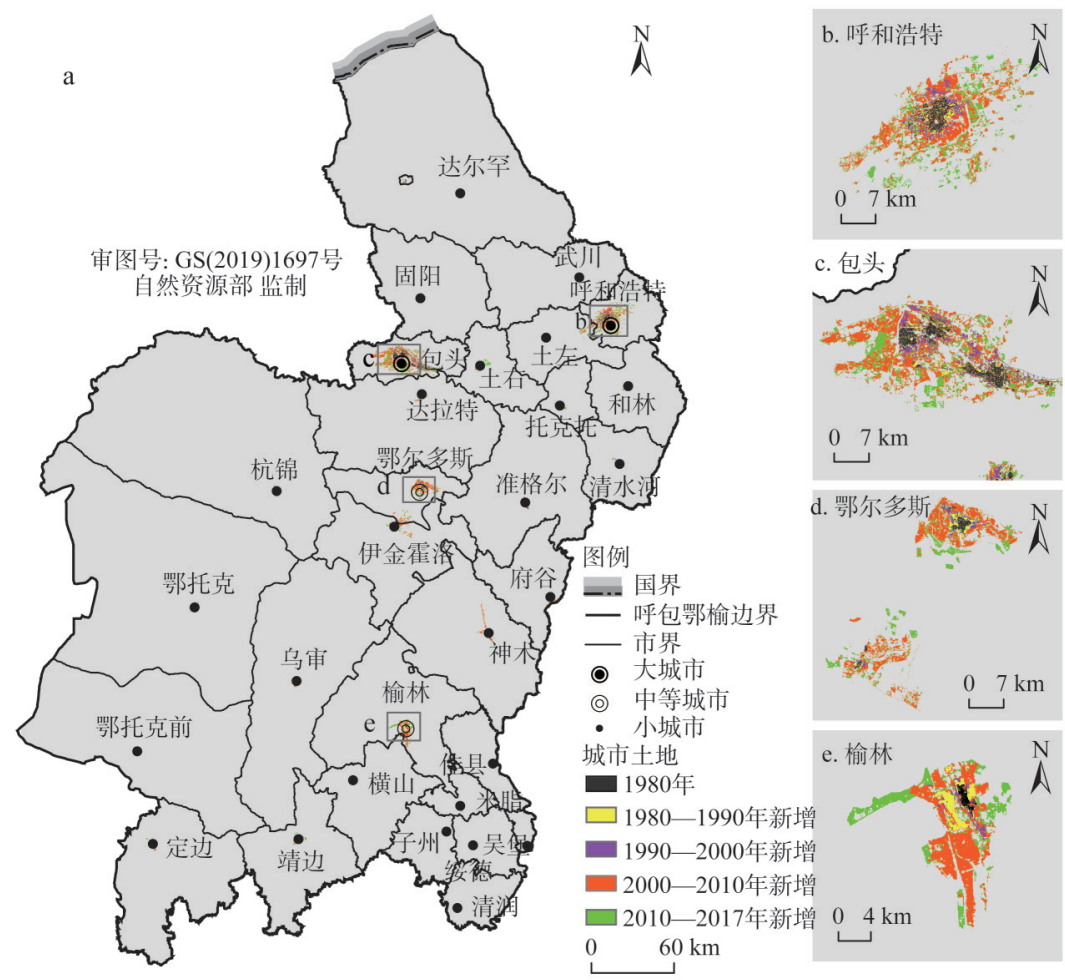

图 4 1980-2017年区域城市土地的空间格局

Fig. 4 The spatial patterns of urban land in the HBOY from 1980 to 2017
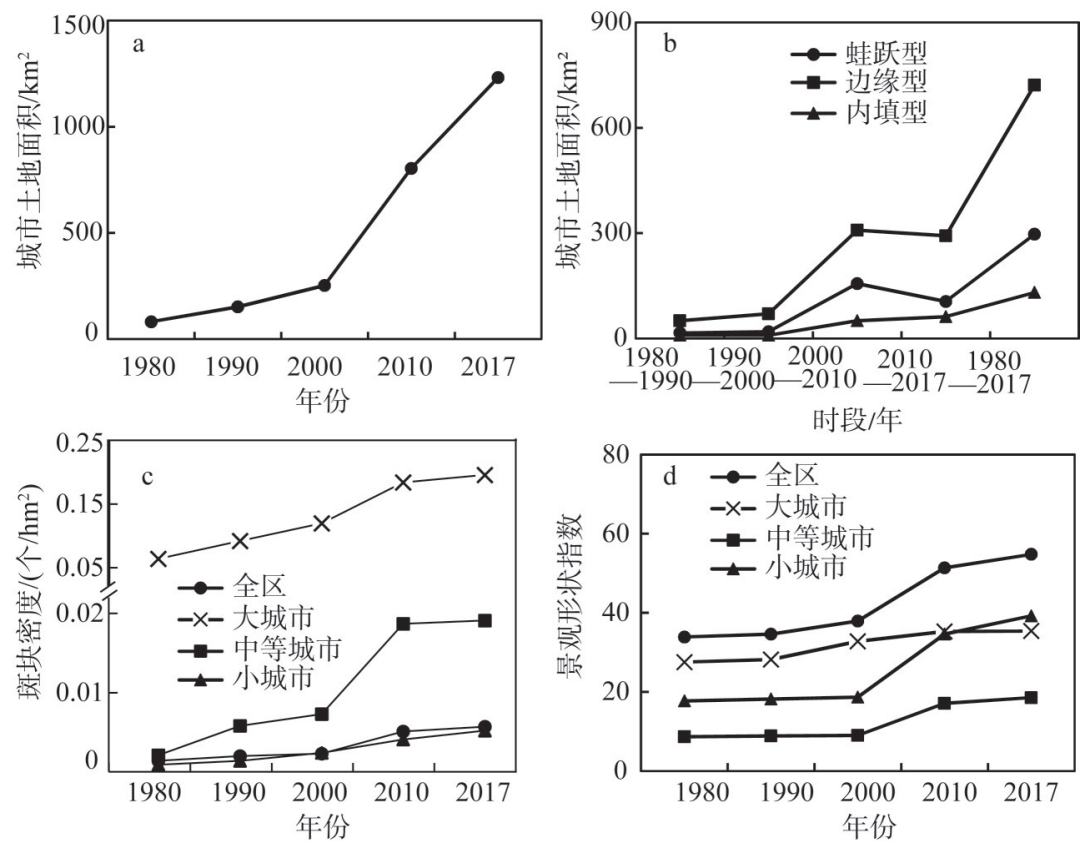

图 5 1980-2017年区域城市扩展过程

Fig. 5 Urban expansion in the HBOY from 1980 to 2017 
表 $21980-2017$ 年区域城市扩展过程

Table 2 Urban expansion in the HBOY from 1980 to 2017

\begin{tabular}{|c|c|c|c|c|c|c|c|c|c|}
\hline \multirow{3}{*}{ 地区 } & \multicolumn{3}{|c|}{ 城市扩展过程 } & \multicolumn{6}{|c|}{ 城市扩展模式 } \\
\hline & \multirow{2}{*}{ 面积 $/ \mathrm{km}^{2}$} & \multirow{2}{*}{$\begin{array}{l}\text { 占全区 } \\
\text { 比例/\% }\end{array}$} & \multirow{2}{*}{$\begin{array}{l}\text { 年均增 } \\
\text { 长率/\% }\end{array}$} & \multicolumn{2}{|c|}{ 边缘型 } & \multicolumn{2}{|c|}{ 蛙跃型 } & \multicolumn{2}{|c|}{ 内填型 } \\
\hline & & & & 面积 $/ \mathrm{km}^{2}$ & 百分比 $/ \%$ & 面积 $/ \mathrm{km}^{2}$ & 百分比 $/ \%$ & 面积 $/ \mathrm{km}^{2}$ & 百分比 $/ \%$ \\
\hline 呼包鄂榆 & 1149.76 & 100 & 7.61 & 721.73 & 62.77 & 296.91 & 25.82 & 131.12 & 11.40 \\
\hline 大城市 & 564.55 & 49.10 & 6.91 & 343.99 & 60.45 & 137.51 & 24.16 & 87.58 & 15.39 \\
\hline 呼和浩特 & 226.12 & 19.67 & 7.39 & 139.60 & 59.00 & 71.35 & 30.15 & 25.67 & 10.85 \\
\hline 包头 & 338.43 & 29.43 & 6.64 & 204.39 & 61.48 & 66.16 & 19.90 & 61.91 & 18.62 \\
\hline 中等城市 & 211.80 & 18.42 & 9.18 & 173.36 & 75.13 & 45.21 & 19.59 & 12.18 & 5.28 \\
\hline 鄂尔多斯 & 120.76 & 10.50 & 8.60 & 96.84 & 72.23 & 32.58 & 24.30 & 4.66 & 3.48 \\
\hline 榆林 & 91.03 & 7.92 & 10.21 & 76.52 & 79.16 & 12.63 & 13.07 & 7.52 & 7.78 \\
\hline 小城市 & 373.41 & 32.48 & 8.25 & 204.38 & 58.41 & 114.19 & 32.63 & 31.36 & 8.96 \\
\hline 土默特左 & 37.93 & 3.30 & 10.31 & 10.98 & 37.30 & 16.01 & 54.38 & 2.45 & 8.32 \\
\hline 托克托 & 15.44 & 1.34 & 11.55 & 10.95 & 70.97 & 3.13 & 20.29 & 1.35 & 8.75 \\
\hline 和林格尔 & 9.63 & 0.84 & 7.22 & 6.04 & 62.59 & 2.80 & 29.02 & 0.81 & 8.39 \\
\hline 清水河 & 3.85 & 0.34 & 8.40 & 2.50 & 63.94 & 1.06 & 27.11 & 0.35 & 8.95 \\
\hline 武川 & 8.73 & 0.76 & 6.36 & 7.14 & 80.50 & 1.17 & 13.19 & 0.56 & 6.31 \\
\hline 土默特右 & 32.21 & 2.80 & 8.06 & 20.63 & 64.09 & 8.38 & 26.03 & 3.18 & 9.88 \\
\hline 固阳 & 8.58 & 0.75 & 6.44 & 6.35 & 72.41 & 1.78 & 20.30 & 0.64 & 7.30 \\
\hline 达茂旗 & 7.92 & 0.69 & 7.61 & 0.98 & 12.36 & 6.67 & 84.11 & 0.28 & 3.53 \\
\hline 达拉特 & 27.83 & 2.42 & 7.20 & 18.81 & 66.94 & 4.38 & 15.59 & 4.91 & 17.47 \\
\hline 准格尔 & 13.86 & 1.21 & 8.60 & 6.00 & 43.01 & 6.80 & 48.75 & 1.15 & 8.24 \\
\hline 鄂托克前 & 7.03 & 0.61 & 6.26 & 4.27 & 60.83 & 1.93 & 27.49 & 0.82 & 11.68 \\
\hline 鄂托克 & 12.30 & 1.07 & 7.60 & 7.09 & 57.83 & 3.60 & 29.36 & 1.57 & 12.81 \\
\hline 杭锦 & 9.86 & 0.86 & 4.05 & 7.32 & 72.69 & 1.43 & 14.20 & 1.32 & 13.11 \\
\hline 乌审 & 11.57 & 1.01 & 6.88 & 6.56 & 56.65 & 4.45 & 38.43 & 0.57 & 4.92 \\
\hline 伊金霍洛 & 35.93 & 3.13 & 10.81 & 11.98 & 49.79 & 11.51 & 47.84 & 0.57 & 2.37 \\
\hline 靖边 & 30.95 & 2.69 & 12.77 & 21.50 & 69.13 & 7.06 & 22.70 & 2.54 & 8.17 \\
\hline 定边 & 20.99 & 1.83 & 8.00 & 14.08 & 67.30 & 5.27 & 25.19 & 1.57 & 7.50 \\
\hline 府谷 & 9.13 & 0.79 & 7.32 & 6.05 & 65.05 & 2.37 & 25.48 & 0.88 & 9.46 \\
\hline 神木 & 36.18 & 3.15 & 11.83 & 15.83 & 42.85 & 17.79 & 48.16 & 3.32 & 8.99 \\
\hline 佳县 & 1.26 & 0.11 & 10.17 & 1.12 & 83.58 & 0.15 & 11.19 & 0.07 & 5.22 \\
\hline 横山 & 11.21 & 0.98 & 10.85 & 3.95 & 64.54 & 1.56 & 25.49 & 0.61 & 9.97 \\
\hline 米脂 & 6.58 & 0.57 & 8.97 & 4.22 & 63.36 & 1.72 & 25.83 & 0.72 & 10.81 \\
\hline 子州 & 3.15 & 0.27 & 6.81 & 2.16 & 69.90 & 0.88 & 28.48 & 0.05 & 1.62 \\
\hline 绥德 & 6.65 & 0.58 & 8.09 & 4.92 & 74.89 & 0.70 & 10.65 & 0.95 & 14.46 \\
\hline 吴堡 & 2.38 & 0.21 & 5.76 & 1.70 & 72.03 & 0.61 & 25.85 & 0.05 & 2.12 \\
\hline 清涧 & 2.27 & 0.20 & 8.41 & 1.25 & 54.35 & 0.98 & 42.61 & 0.07 & 3.04 \\
\hline
\end{tabular}

包头城市土地扩展面积最大，为 $338.43 \mathrm{~km}^{2}$ ，占全区城市土地扩展总面积的 $29.43 \%$ （表 2)。 同期，区域城市扩展过程以边缘型为主。在全区尺度上，边缘型城市扩展面积为 $721.73 \mathrm{~km}^{2}$, 占城市扩展总面积的 $62.77 \%$ 。蛙跃型和内填型城市扩展面积分别为 $296.91 \mathrm{~km}^{2}$ 和 $131.12 \mathrm{~km}^{2}$ ，分别占城市扩展总面积的 $25.82 \%$ 和 $11.40 \%$ （图 $5 \mathrm{~b}$ 、表 2 ）。对于不同规模 
等级城市, 城市扩展过程均以边缘型为主。其中, 大城市边缘型扩展面积最大, 为 $343.99 \mathrm{~km}^{2}$, 占大城市扩展总面积的 $60.45 \%$ （表 2)。在大城市中, 包头边缘型扩展面积 最大, 为 $204.39 \mathrm{~km}^{2}$, 占包头城市扩展总面积的 $61.48 \%$ (表2）。

区域 1980-2017 年间城市景观越来越破碎，形状越来越复杂。在全区尺度上，斑块 密度在 1980-2017 年之间呈现不断增长趋势, 由 0.0014 增长至 0.0057 。对于大中小城 市, 斑块密度均呈现增加趋势, 其中大城市增加最多, 由 0.0639 增加到 0.1957 (图 5c)。 同时，在全区尺度上，城市景观的形状指数在 1980-2017年之间也不断增加，由 33.88 增长至 54.78。对于大中小城市, 景观形状指数都呈现增加趋势, 其中小城市增加最多, 由 17.72 增加到 39.23 (图 5d)。

\section{2 区域城市扩展的区位因素}

在全区尺度上, 到城市中心距离的重要性最高, 为 $42.62 \%$ 。国道、高速公路和铁路 等交通因素的重要性也比较高，分别为 $16.20 \% 、 14.15 \%$ 和 $11.80 \%$ 。不同规模等级城市的 区位因素重要性存在明显差异。对于大城市, 到城市中心距离、高程和干燥度都比较重 要, 重要性分别为 $21.27 \% 、 20.33$ 和 $20.23 \%$; 其次国道和河流的影响也比较大, 分别为 $8.73 \%$ 和 $8.12 \%$ 。对于中等城市, 到城市中心距离影响最大, 为 $46.71 \%$; 国道、铁路、高 速公路等交通因素和到河流距离都比较重要，重要性分别为 $18.09 \% 、 12.08 \% 、 8.13 \%$ 和 $8.15 \%$ 。对于小城市，到城市中心距离重要性最高，为 $53.37 \%$; 国道、高速公路和铁路 等交通因素的重要性也比较高，分别为 $11.34 \% 、 10.03 \%$ 和 $9.89 \%$ （图 6a)。总体来看，到 城市中心距离在区域 1980-2017年城市扩展过程中扮演了最重要的角色。其次，交通因 素、高程、干燥度与到河流距离等区位因素的影响也比较大。

区位因素对区域城市扩展过程的影响都存在尺度效应，其中地形、干燥度和到河流 距离因素的尺度效应相对比较明显。高程、坡度和坡向等地形因素对大中小城市的扩展 过程影响分别为 $27.17 \% 、 2.18 \%$ 和 $6.96 \%$, 其对大城市的影响是对小城市的 4.02 倍。干燥 度对大中小城市的影响分别为 $20.23 \% 、 4.65 \%$ 和 $5.17 \%$, 其对大城市的影响是对小城市的 3.91 倍。到河流距离对大中小城市的影响分别为 $8.12 \% 、 8.15 \%$ 和 $3.44 \%$, 其对大城市的 影响是对小城市的 2.36 倍。此外, 到城市中心距离和交通因素, 其对大城市的影响分别 是对小城市的 0.40 倍和 0.74 倍（图 6a)。总体来看, 地形因素对不同规模等级城市扩展过 程影响的尺度效应最明显, 其次是干燥度和到河流距离因素。

城市扩展的区位因素重要性在不同时间段存在明显的变化。国道和高速公路因素对 新增城市土地影响在 2010-2017 年间最大，分别为 16.46\% 和 13.95\%，为其在 1990 2000 年间重要性的 1.43 倍和 1.58 倍（图 6b)。铁路在 2000-2010年间对城市扩展影响最 高, 为 17.01\%, 是其在 1990-2000年影响的 1.87倍（图 6b)。此外, 高程、干燥度和河 流等因素在不同时期对新增城市的影响呈现递减趋势，其重要性分别下降了 $52.85 \%$ 、 $63.93 \%$ 和 $70.82 \%$ (图 6b)。

对于不同城市扩展模式, 到城市中心距离均是最重要的区位因素。对于蛙跃型、边缘型 和内填型城市扩展，到城市中心距离的重要性分别为 19.52\%、29.99\%和 $14.73 \%$ （图 7a）。 其次河流和交通因素也比较重要。其中, 到河流距离对三种不同扩展模式的影响分别为 $14.79 \%$ 、9.59\%和 $14.39 \%$ 。交通因素的重要性分别达到 $38.07 \%$ 、39.80\%和 $40.45 \%$ （图 7a）。

区位因素对于不同扩展模式的重要性随时间的变化而变化。对于蛙跃型扩展，到城 

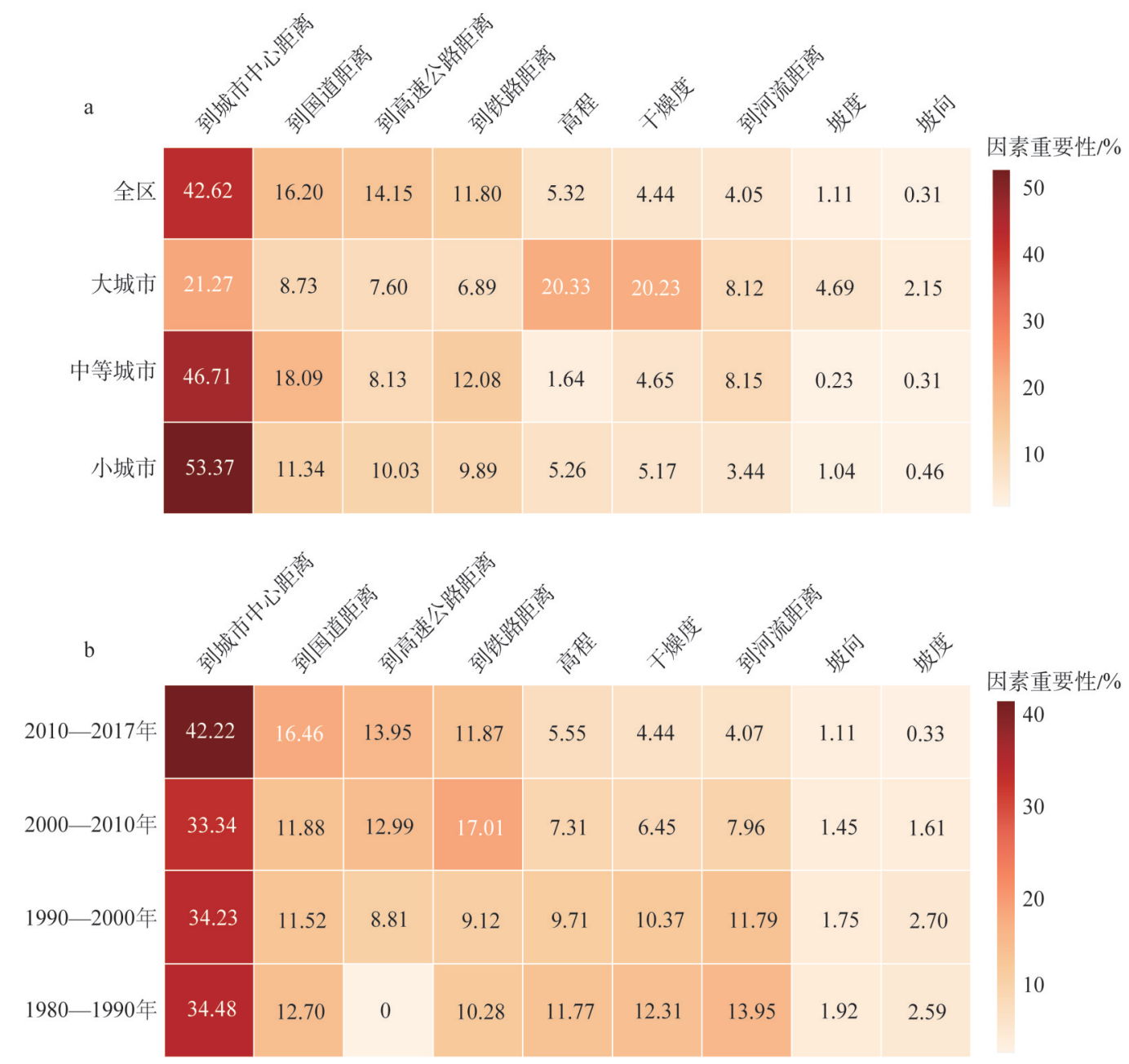

注： a为 1980-2017年区位因素的重要性； b 为全区不同时间段区位因素的重要性。

图6 区位因素的重要性

Fig. 6 The importance of location factors

市中心距离的重要性在 1990-2017 年间呈增长趋势，由 18.64\%增长到 $31.43 \%$ 。干燥度 和高程因素的重要性呈减少趋势，分别由 8.93\%和 15.25\%下降到 3.03\%和 13.73\%（图 7b)。 对于边缘型扩展，1990-2017年间，到城市中心距离对区域城市扩展的影响呈下降趋 势, 由 $33.85 \%$ 下降到 $28.07 \%$ 。高程和到河流距离影响呈现增加趋势, 分别由 $7.77 \%$ 和 9.51\%增长到 $12.42 \%$ 和 13.14\%（图 7b）。对于内填型扩展，国道、高速公路和铁路等交 通因素的重要性程序增长趋势，由 29.07\%增长到38.70\%。到城市中心距离在1990-2017年 间呈下降趋势，由 $35.01 \%$ 下降到 $24.13 \%$ （图 7b)。

\section{3 讨论}

\section{1 城市土地数据可靠性评价}

本文中目视解译获取的城市土地数据能够准确地反映区域城市土地的实际状况。首 先, 参考相关研究 ${ }^{[33,34]}$, 分别利用区域 GDP 年均增长量和城镇人口年均增长量与城市土 


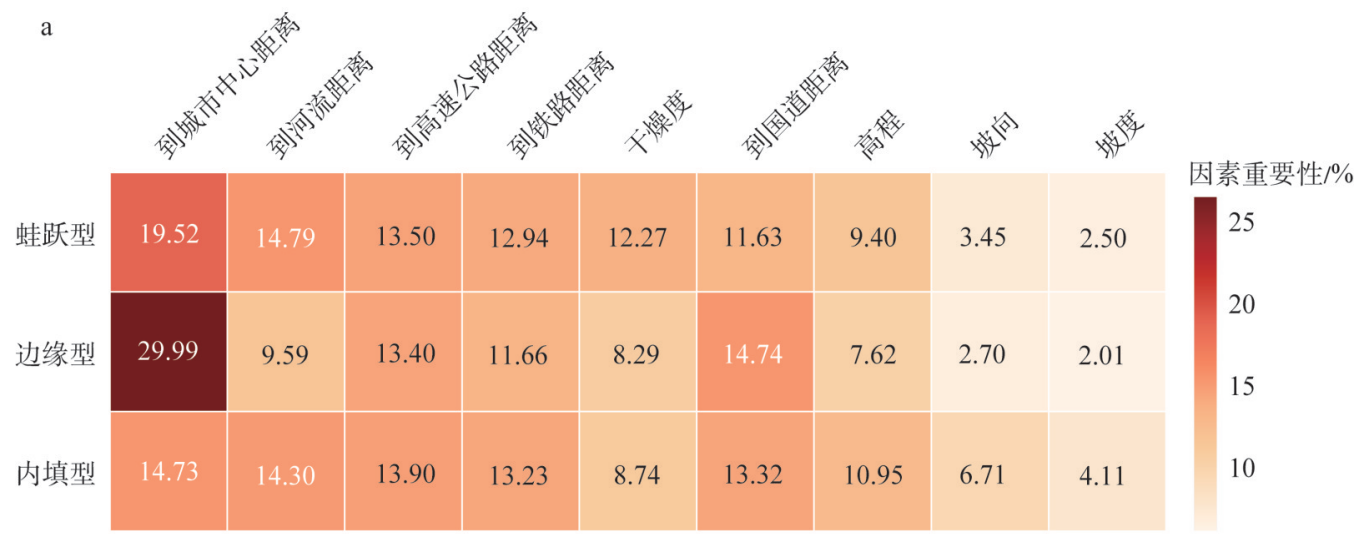

b

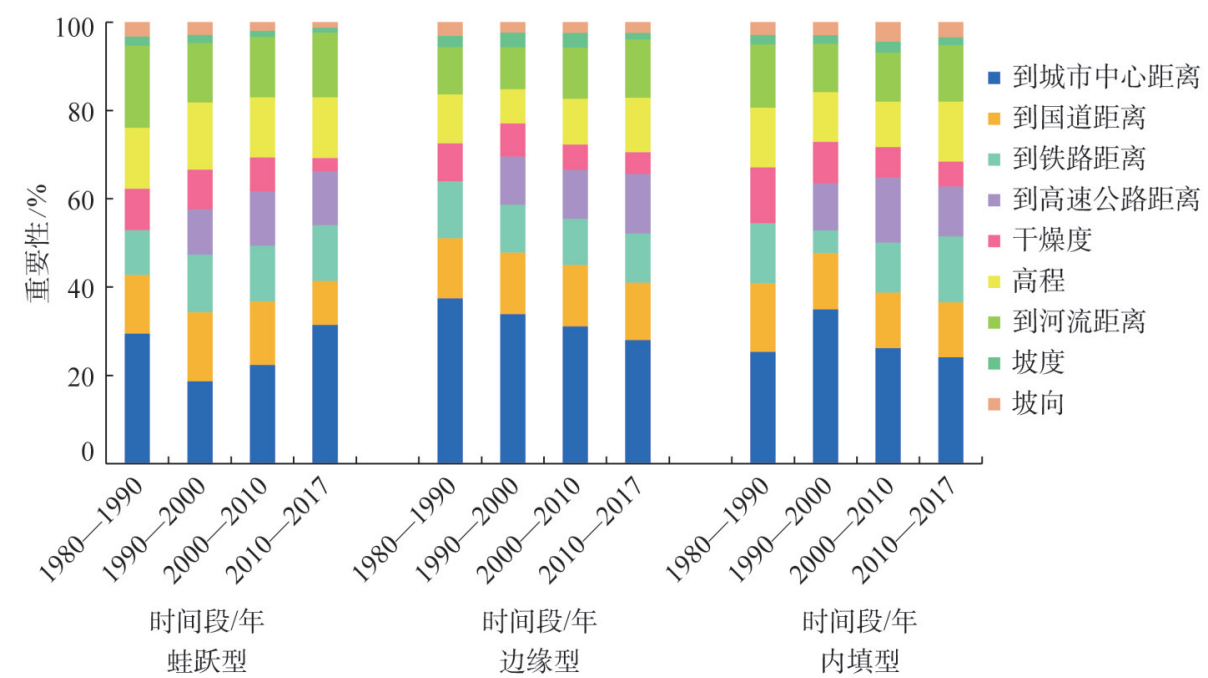

注： $\mathrm{a}$ 为 1980-2017年区位因素的重要性； b 为不同时间段区位因素的重要性。

图 7 不同城市扩展模式的区位因素重要性

Fig. 7 The importance of location factors on urban growth modes

地年均增长量进行相关分析。结果显示本文提取的城市土地信息与区域GDP 和城镇人口 数据有显著相关性, 相关系数分别为 0.85 和 0.96 , 都通过了 0.001 的显著性检验（图 8)。 其次, 采用等量分层随机抽样 (Equalized stratified random sampling) 的方法选取 1000 个 样本点, 结合 Google Earth 高分辨率 $(4 \mathrm{~m})$ 影像计算误差矩阵。结果显示, 1980 年、 1990 年、 2000 年、 2010 年和 2017 年的城市土地数据 Kappa 系数分别为 $0.86 、 0.87$ 、 $0.87 、 0.85$ 和 0.86 , 总体精度分别为 $92.80 \% 、 93.30 \% 、 93.40 \% 、 92.60 \%$ 和 $92.80 \%$ 。此 外，以 1990-2010年的数据为例，将本文获取的城市土地数据与已有的长时间序列且同 分辨率的数据 ${ }^{[28]}$ 进行对比发现, 本文的城市土地数据明显地减少了漏分和错分, 更符合 区域城市土地的实际状况（图9）。

\section{2 随机森林方法比 Logistic 回归更有利于揭示区位因素的基本特征}

ROC (Receiver operating characteristic) 曲线和 AUC (Area under curve) 指标是判 断机器学习二分类预测模型优劣的常用方法, AUC 值越大表示该算法精度越高 ${ }^{[1,3,35]}$ 。为 此，基于相同的区位因素，分别利用随机森林和Logistic 回归方法模拟区域 1980-2017年 

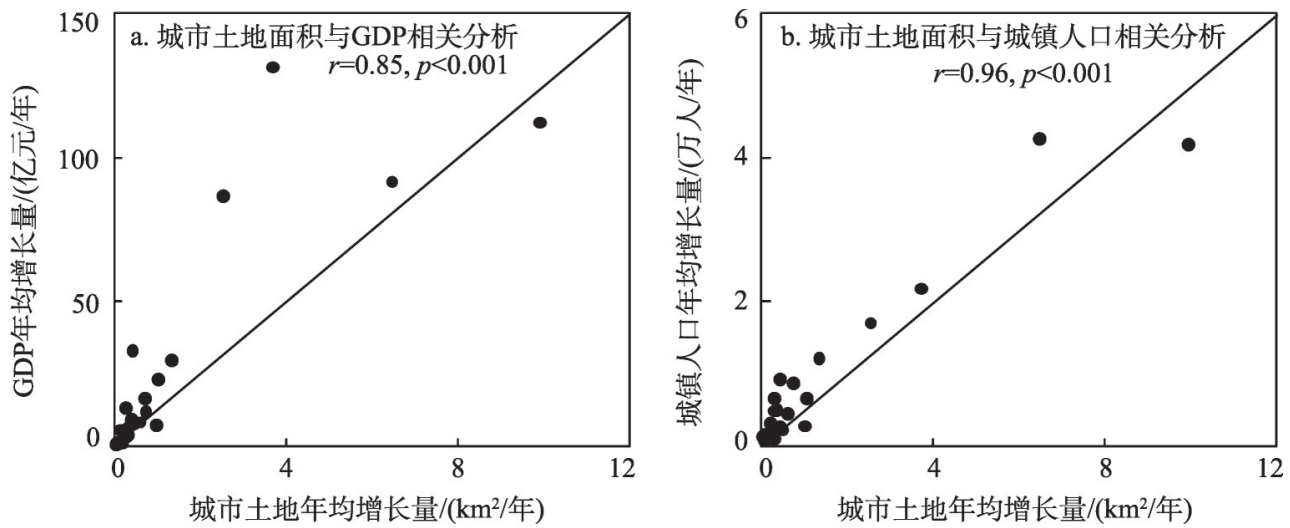

图 8 1980-2017年区域城市土地数据的数量精度验证

Fig. 8 The quantitative accuracy assessment of urban land in the HBOY from 1980 to 2017

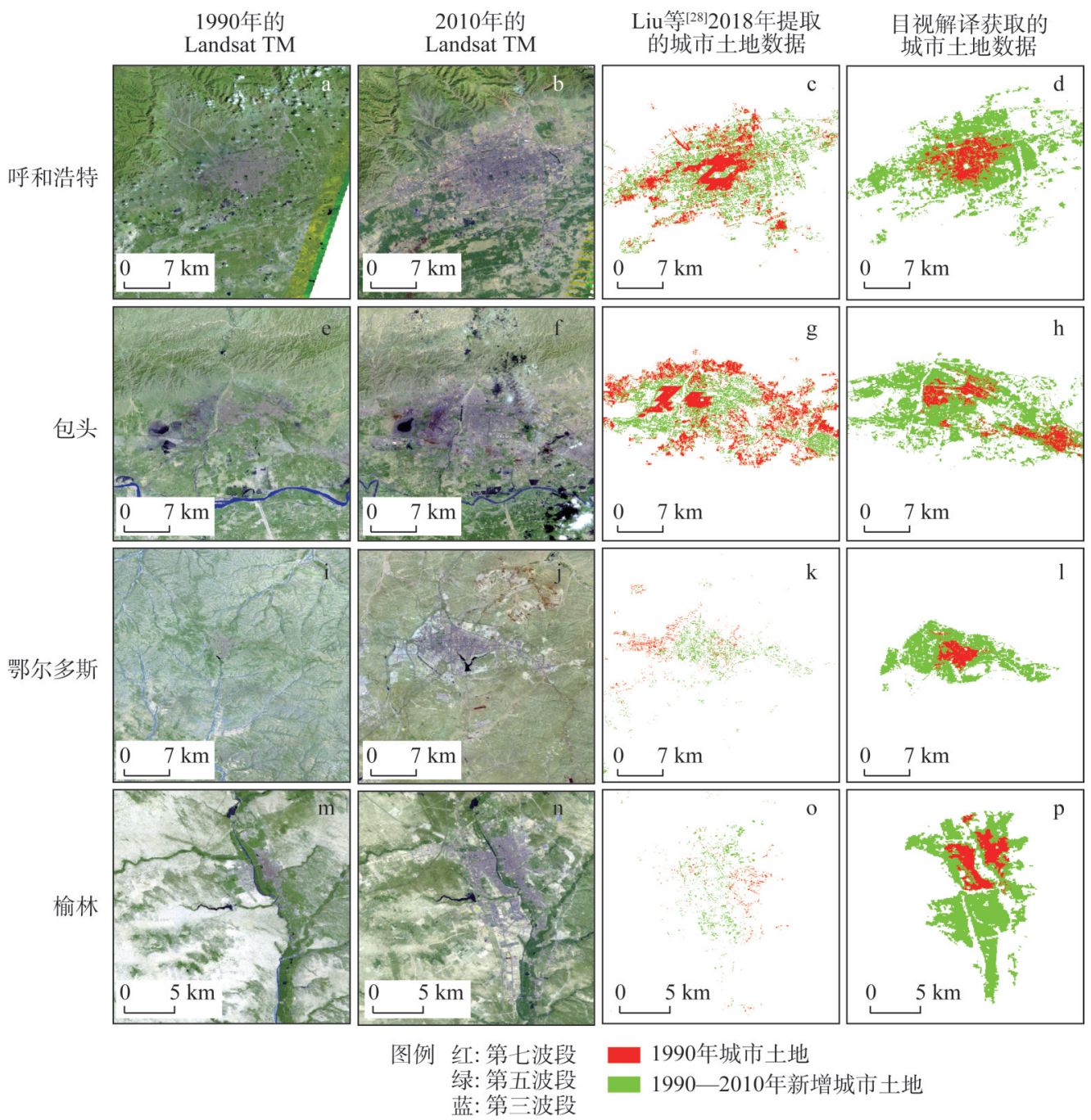

图 9 目视解译获取的区域 1990-2010年城市土地数据与已有数据对比

Fig. 9 The comparison between the urban land data obtained by visual interpretation and the existing data from 1990 to 2010 
的城市扩展过程。通过与实际的城市 土地数据对比, 随机森林方法的 AUC 值为 0.97 , 而 Logistic 回归的 AUC 值为 0.88（图 10), 表明随机森林方法在呼 包鄂榆城市群的实践应用上表现更 好, 精度更高。这主要是因为随机森 林方法更适宜于解决非线性问题。一 方面, 相较于 Logistic 回归, 随机森林 可以处理非线性特征, 而且考虑了各 特征变量之间的相互作用, 能够很好 地解决特征变量之间的多重共线性影 响 ${ }^{[18,35]}$ 。另一方面, 随机森林模型在数 据训练的过程中采用有放回的随机抽 样, 同时在选取特征变量是也是随机 选取，这样保证了决策树之间的独立 性，提高了模型的模拟能力 ${ }^{[18,36]}$ 。

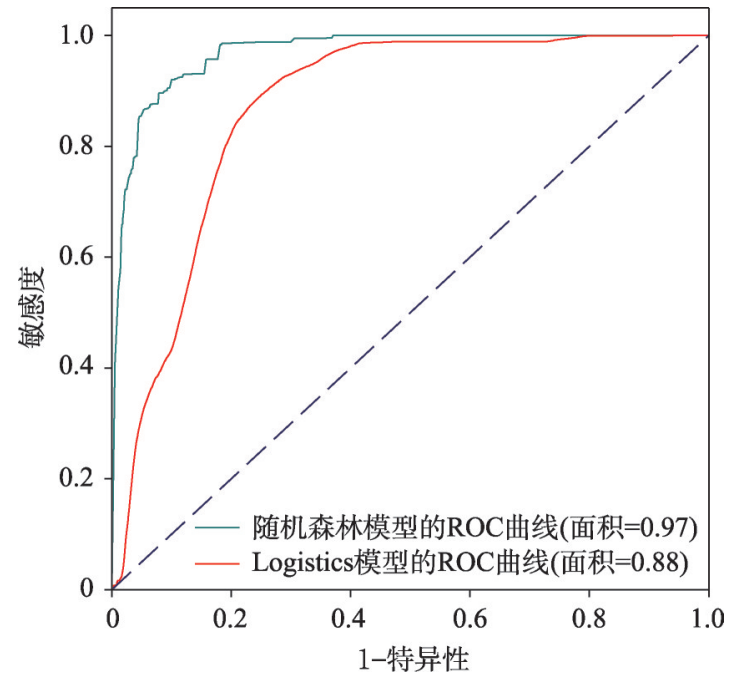

图 10 随机森林和Logistic 回归的 ROC 曲线

Fig. 10 The ROC curves of random forest and logistic regression

\section{4 结论}

随机森林方法能够更加有效地量化旱区城市扩展过程区位因素的基本特征。在呼包 鄂榆城市群案例分析中, 随机森林方法的 AUC 值达到了 0.97, 明显高于Logistic 回归方 法的 AUC 值。其次, 到城市中心距离对区域 1980-2017年城市扩展过程影响最大, 重 要性为 $42.62 \%$ 。此外, 区位因素对区域城市扩展过程的影响都存在尺度效应, 其中高 程、坡度和坡向等地形因素、气候以及到河流距离的尺度效应最为明显。

为此, 建议在未来的城市规划和建设中，区域上应继续高度重视地形、气候和河流 等自然要素的制约作用。对于大城市，应结合区域高精度的地形数据制定城市规划，同 时应该将气候条件纳人城市规划中，充分考虑气候对新增城市土地的制约影响。此 外，也需要考虑河流对新增城市土地的影响。比如，在制定包头市国土空间总体规划 （2021-2035 年）的过程中, 应将地形、干燥度和到河流距离作为优先考虑的限制条 件，引导城市向地形条件相对较好、水资源丰富以及相对湿润的南部扩展。对于中等城 市，应主要依托交通网络制定城市规划，充分发挥交通因素对城市的带动作用。比如， 鄂尔多斯市未来城市建设应充分考虑呼包鄂榆城市群规划 “三纵三横” 交通网络中 “鄂 一准一乌交通运输大通道” 和 “包茂综合运输大通道” 的影响, 借助城市群综合交通运 输网带动区域城市发展 ${ }^{[23]}$ 。对于小城市, 需要紧密依托现有的城市格局, 充分发挥老城 区的辐射带动作用。此外，在未来的城市规划中对于不同模式的城市扩展也应该采取针 对性的措施。事实上，旱区的生态环境脆弱，气候和河流等自然因素对城市发展的制约 作用比湿润地区更明显 ${ }^{[37,38]}$ 。对旱区的城市发展, 应该保持高度的谨慎态度, 特别要注意 在水资源承载力和地形的约束范围内进行，对不同规模等级城市和扩展模式针对性地制 定规划，因地制宜地进行城市建设。

本文运用随机森林方法定量分析了区位因素对旱区不同规模等级城市扩展过程的影 
响，并提出相应的政策建议，这对旱区城市可持续发展具有重要意义。但本文也存在一 些不足。首先，未考虑周边区位因素对目标像元的影响。目标像元是否转化为城市像 元，不仅受该像元处的区位因素影响，往往还受到一定邻域范围内的区位因素影响 ${ }^{[6]}$ 。其 次，本文只分析了区位因素对城市扩展的影响。城市扩展过程是一个极其复杂的过程， 受到政策规划、社会经济发展和微观区位等多方面因素的影响 ${ }^{[8,38]}$ 。在未来的研究中，可 以使用深度卷积神经网络，通过设置卷积核参数来分析目标像元一定邻域范围内区位因 素对城市扩展过程的影响。此外，可以整合社会经济、规划政策以及地理区位等多因素 综合分析旱区城市扩展的驱动机制。

\section{参考文献(References):}

[1] Millennium Ecosystem Assessment. Ecosystems and Human Well-being. Synthesis. Vol. 42. Washington DC: Island Press, 2005: 29.

[2] BAI X M, CHEN J, SHI P J. Landscape urbanization and economic growth in China: Positive feedbacks and sustainability dilemmas. Environmental Science \&Technology, 2012, 46(1): 132-139.

[3] CLARKE K C, HOPPEN S, GAVDOS L J. A self-modified cellular automaton model of historical urbanization in the San Francisco Bay Area. Environment and Planning B, 1997, (24): 247 -261.

[4] 摆万奇, 赵士洞. 土地利用变化驱动力系统分析. 资源科学, 2001, 23(3): 39-41. [BAI W Q, ZHAO S D. An analysis on driving force system of land use changes. Resources Science, 2001, 23(3): 39-41.]

[5] 方创琳, 宋吉涛, 萄雪芹. 中国城市群可持续发展理论与实践. 北京: 科学出版社, 2010: 31-36. [FANG C L, SONG J T, LIN X Q. Theory and Practice of Sustainable Development of Urban Agglomerations in China. Beijing: Science Press, 2010: 31-36.]

[6] HE C Y, LI J W, ZHANG X L, et al. Will rapid urban expansion in the drylands of Northern China continue: A scenario analysis based on the land use scenario dynamics- urban model and the shared socioeconomic pathways. Journal of Cleaner Production, 2017, 165: 57-69.

[7] HE C Y, LIU Z F, GOU S Y, et al. Detecting global urban expansion over the last three decades using a fully convolutional network. Environmental Research Letters, 2019, 14(3), Doi: 10.1088/1748-9326/aaf936.

[8] HE C Y, LIU Z F, TIAN J, et al. Urban expansion dynamics and natural habitat loss in China: A multiscale landscape perspective. Global Change Biology, 2014, 20(9): 2886-2902.

[9] MCDONALD R I, KAREIVA P, FORMAN R T T. The implications of current and future urbanization for global protected areas and biodiversity conservation. Biological Conservation, 2008, 141(6): 1695-1703.

[10] LIU Z F, DING M H, HE C Y, et al. The impairment of environmental sustainability due to rapid urbanization in the dryland region of Northern China. Landscape and Urban Planning, 2019, 187: 165-180.

[11] 蒋祺, 郑伯红. 城市用地扩展对长沙市水系变化的影响. 自然资源学报, 2019, 34(7): 1429-1439. [JIANG Q, ZHENG B H. The relationship between the change of water system and the urban land expansion in Changsha. Journal of Natural Resources, 2019, 39(7): 1429-1439.]

[12] 宋世雄, 刘志锋, 何春阳, 等. 城市扩展过程对自然生境影响评价的研究进展. 地球科学进展, 2018, 33(10): 10941104. [SONG S X, LIU Z F, HE C Y, et al. Research progress on assessing the impacts of urban expansion on natural habitats. Advances in Earth Science, 2018, 33(10): 1094-1104.]

[13] 潮洛濛, 翟继武, 韩倩倩. 西部快速城市化地区近 20 年土地利用变化及驱动因素分析: 以呼和浩特市为例. 经济地 理, 2010, 30(2): 239-243. [CHAO L M, ZHAI J W, HAN Q Q. Urban expand and driving factors in the recent 20 years in case of Huhhot city. Economic Geography, 2010, 30(2): 239-243.]

[14] 李骞国, 石培基, 魏伟. 干旱区绿洲城市扩展及驱动机制: 以张掖市为例. 干旱区研究, 2015, 32(3): 598-605. [LI Q G, SHI P J, WEI W. Research on urban expansion and drive mechanism in an oasis town of arid region: A case of Zhangye city. Arid Zone Research, 2015, 32(3): 598-605.]

[15] 黄庆旭, 何春阳, 史培军, 等. 城市扩展多尺度驱动机制分析: 以北京为例. 经济地理, 2009, 29(5): 714-721. 
[HUANG Q X, HE C Y, SHI P J, et al. Understanding multi-scale urban expansion driving forces: In the case study of Beijing. Economic Geography, 2009, 29(5): 714-721.]

[16] SHAFIZADEH-MOGHADAM H, HELBICH M. Spatiotemporal variability of urban growth factors: A global and local perspective on the megacity of Mumbai. International Journal of Applied Earth Observation and Geoinformation, 2015, 35: 187-198.

[17] WU W J, ZHAO S Q, HENEBRY G M. Drivers of urban expansion over the past three decades: A comparative study of Beijing, Tianjin, and Shijiazhuang. Environmental Monitoring and Assessment, 2019, 191: 34.

[18] BREIMAN L. Random forests. Machine Learning, 2001, 45: 5-32.

[19] KAMUSOKO C, GAMBA J. Simulating urban growth using a Random Forest-Cellular Automata (RF-CA) Model. ISPRS International Journal of Geo-Information, 2015, 4(2): 447-470.

[20] ZHANG D C, LIU X P, WU X Y, et al. Multiple intra-urban land use simulations and driving factors analysis: A case study in Huicheng, China. GIScience \& Remote Sensing, 2018, Doi: 10.1080/15481603.2018.1507074.

[21] 孙泽祥, 刘志锋, 何春阳, 等. 中国快速城市化干燥地区的生态系统服务权衡关系多尺度分析: 以呼包鄂榆地区为 例. 生态学报, 2016, 36(15): 4881-4891. [SUN Z X, LIU Z F, HE C Y, et al. Multi-scale analysis of ecosystem service trade-offs in urbanizing drylands of China: A case study in the Hohhot-Baotou-Ordos-Yulin region. Acta Ecologica Sinica, 2016, 36(15): 4881-4891.]

[22] HUANG Q X, MENG S T, HE C Y, et al. Rapid urban land expansion in earthquake-prone areas of China. International Journal of Disaster Risk Science, 2019, 10(1): 43-56.

[23] 国家发改委. 呼包鄂榆城市群发展规划. www. gov. cn/xinwen/2018-03/07/content_5271788. htm, 2019-08-04. [National Development and Reform Commission. The plan for Hohhot-Baotou-Ordos-Yulin cluster. www. gov. cn/xinwen/ 2018-03/07/content_5271788.htm, 2019-08-04.]

[24] LIU Z F, VERBURG P H, WU J G, et al. Understanding land system change through scenario-based simulations: A case study from drylands in Northern China. Environmental Management, 2017, 59: 440-454.

[25] 丁美慧, 刘志锋, 何春阳, 等. 中国北方农牧交错带城市扩展过程对植被净初级生产力影响研究: 以呼包鄂地区为 例. 干早区地理, 2017, 40(3): 614-621. [DING M H, LIU Z F, HE C Y, et al. Impacts of urban expansion on net primary productivity in the agro-pastoral ecotone in Northern China: A case of Hohhot-Baotou-Ordos region. Arid Land Geography, 2017, 40(3): 614-621.]

[26] 孙泽祥, 刘志锋, 何春阳, 等. 中国北方干燥地区城市扩展过程对生态系统服务的影响: 以呼和浩特一包头一鄂尔 多斯城市群地区为例. 自然资源学报, 2017, 32(10): 1691-1704. [SUN Z X, LIU Z F, HE C Y, et al. Impacts of urban expansion on ecosystem services in the drylands of Northern China: A case study in the Hohhot-Baotou-Ordos Urban Agglomeration region. Journal of Natural Resources, 2017, 32(10): 1691-1704.]

[27] SONG S X, LIU Z F, HE C Y, et al. Evaluating the effects of urban expansion on natural habitat quality by coupling localized shared socioeconomic pathways and the land use scenario dynamics-urban model. Ecological Indicators, 2020, 112, Doi: 10.1016/j.ecolind.2020.106071.

[28] LIU X P, HU G H, CHEN Y M, et al. High-resolution multi-temporal mapping of global urban land using Landsat images based on the Google Earth Engine Platform. Remote Sensing of Environment, 2018, 209: 227-239.

[29] 董宁, 韩兴国, 邬建国. 内蒙古鄂尔多斯市城市化时空格局变化及其驱动力. 应用生态学报, 2012, 23(4): 1097-1103. [DONG N, HAN X G, WU J G. Changes in the spatiotemporal pattern of urbanization in Erdos of Inner Mongolia and related driving forces. Chinese Journal of Applied Ecology, 2012, 23(4): 1097-1103.]

[30] LIU X P, LI X, CHEN Y M. A new landscape index for quantifying urban expansion using multi-temporal remotely sensed data. Landscape Ecology, 2010, 25: 671-682.

[31] 李春林, 刘沝, 胡远满, 等. 基于增强回归树和 Logistic 回归的城市扩展驱动力分析. 生态学报, 2014, 34(3): 727737. [LI C L, LIU M, HU Y M, et al. Driving forces analysis of urban expansion based on boosted regression trees and Logistic regression. Acta Ecologica Sinica, 2014, 34(3): 727-737.]

[32] 翟涌光, 屈忠义, 吕萌. 西北部少数民族地区城市扩展特征分析: 以呼和浩特市为例. 测绘科学, 2020, 45(4): 97104, 124. [ZHAI Y G, QU Z Y, LYU M. Analysis of urban expansion in northwest minority area: A case study of Hohhot city. Science of Surveying and Mapping, 2020, 45(4): 97-104, 124.] 
[33] LIU Z F, HE C Y, ZHANG Q F, et al. Extracting the dynamics of urban expansion in China using DMSP-OLS nighttime light data from 1992 to 2008. Landscape and Urban Planning, 2012, 106(1): 62-72.

[34] XU M, HE C Y, LIU Z F, et al. How did urban land expand in China between 1992 and 2015? A multi-scale landscape analysis. PLoS One, 2016, 11(5): 1-19.

[35] PONTIUS R G JR, SCHNEIDER L C. Land-cover change model validation by an ROC method for the Ipswich watershed, Massachusetts, USA. Agriculture, Ecosystems and Environmet, 2001, 85: 239-248.

[36] BIAU G. Analysis of a random forests model. Journal of Machine Learning Research, 2012, 13: 1063-1095.

[37] LI G D, SUN S A, FANG C L. The varying driving forces of urban expansion in China: Insights from a spatial-temporal analysis. Landscape and Urban Planning, 2018, 174: 63-77.

[38] 曹小曙. 基于人地耦合系统的国土空间重塑. 自然资源学报, 2019, 34(10): 2051-2059. [CAO X S. Geogovernance of national land use based on coupled human and natural systems. Journal of Natural Resources, 2019, 34(10): 2051-2059.]

\title{
Study on the location factors of urban expansion in the drylands: A case study in the Hohhot-Baotou-Ordos-Yulin Urban Agglomeration, China
}

\author{
SONG Shi-xiong ${ }^{1,2}, Z_{\text {ZHANG Jin-xi }}^{1,2}$, LIU Zhi-feng ${ }^{1,2}$, HE Chun-yang ${ }^{1,2}$ \\ (1. Center for Human-Environment System Sustainability (CHESS), State Key Laboratory of Earth Surface \\ Processes and Resource Ecology (ESPRE), Beijing Normal University, Beijing 100875, China; 2. School of \\ Natural Resources, Faculty of Geographical Science, Beijing Normal University, Beijing 100875, China)
}

\begin{abstract}
Understanding the characteristics of location factors of urban expansion is important to urban sustainable development in the drylands. Taking the Hohhot-Baotou- Ordos- Yulin Urban Agglomeration in China as an example, this study assessed the impacts of location factors on urban expansion using the random forest model. The results indicated that the random forest model can effectively quantify the basic characteristics of location factors of urban expansion in the drylands, with the area under curve (AUC) index of 0.97. The distance to the urban centers had the greatest influence on urban expansion from 1980 to 2017, with the importance of $42.62 \%$. The traffic factors such as highways, national roads, and railways also played an important role with the importance above $10 \%$. All location factors had scaling effects on regional urban expansion, among which the topographic, the climate and the river factors had the most obvious effects. The importance of topography, climate and rivers to large cities was $27.17 \%, 20.23 \%$ and $8.12 \%$, respectively, 4.02 times, 3.91 times and 2.36 times that of small cities. Thus, we suggest that the local governments should pay close attention to the constraint effects of the natural factors (e.g., topography, climate and rivers) on the urban construction in the drylands, and perform urban planning and construction according to local conditions.
\end{abstract}

Keywords: random forest; location factor; urban expansion; drylands; Hohhot-Baotou-OrdosYulin Urban Agglomeration 